\title{
Transcriptome Profile Analysis of Twisted Leaf Disease Response in Susceptible Sugarcane with Narenga porphyrocoma Genetic Background
}

\author{
Jinju Wei ${ }^{1} \cdot$ Zhihui Xiu $^{2}$ (D) Huiping Ou ${ }^{1} \cdot$ Junhui Chen ${ }^{2} \cdot$ Huayan Jiang ${ }^{2} \cdot$ Xiaoqiu Zhang $^{1} \cdot$ Ronghua Zhang $^{1}$. \\ Hui Zhou ${ }^{1}$ - Yiyun Gui ${ }^{1} \cdot$ Haibi $\mathrm{Li}^{1} \cdot$ Yangrui $\mathrm{Li}^{1} \cdot$ Rongzhong Yang $^{1} \cdot$ Dongliang Huang ${ }^{1} \cdot$ Hongwei Tan $^{1} \cdot$ Xihui Liu $^{1}$
}

Received: 10 January 2019 / Accepted: 9 May 2019/Published online: 15 June 2019

(C) The Author(s) 2019

\begin{abstract}
Sugarcane is an important industrial crop with a high sugar yield that has become a leading energy crop worldwide. It is widely cultivated in tropical and subtropical regions. Various diseases beset the cultivation of sugarcane. The molecular study of disease resistance in sugarcane is limited by its complicated genome. In our study, RNA-seq was employed to detect the mechanism of twisted leaf disease tolerance in modern cultivar sugarcane, which derived from Narenga porphyrocoma. We completed highthroughput transcriptomic sequencing of 12 samples, including three stages of a susceptible (NSBC1_T "H3-8") and an unsusceptible cultivar (NSBC1_CK "H-19") with two biological repeats, respectively. Using the Saccharum spontaneum genome as reference, the average mapping ratio of the clean data was over $70 \%$. Among the differentially expressed genes between H3-8 and H3-19, we focused on the analysis of hormone pathways and resistance $(R)$ genes. The results showed that twisted leaf disease triggers hormone networks and around $40 \%$ of $R$ genes conditioned lower expression in the susceptible cultivar. One of the possible reasons for $\mathrm{H} 3-8$ being susceptible to twisted leaf disease might be the null/retarded response of $R$ genes, especially in pre-onset stage (46\% down-regulated) of pathogens infection.
\end{abstract}

Keywords Hormones $\cdot R$ genes $\cdot$ RNA-seq $\cdot$ Sugarcane

Jinju Wei, Zhihui Xiu and Huiping Ou equally contributed to this work.

Communicated by: Rui Xia

Electronic supplementary material The online version of this article (https://doi.org/10.1007/s12042-019-09231-5) contains supplementary material, which is available to authorized users.

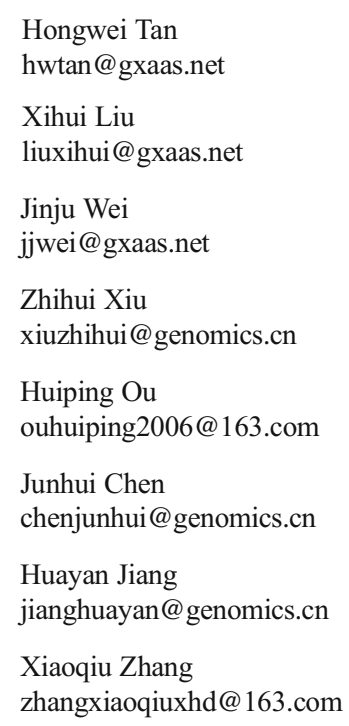




\section{Abbreviations \\ NR Non-redundant protein sequences in NCBI \\ $\mathrm{C} \mathrm{O} \mathrm{G} \mathrm{/} \mathrm{Clusters} \mathrm{of} \mathrm{orthologous} \mathrm{group}$ \\ KOG \\ KEGG Kyoto encyclopedia of genes and genomes databases \\ TGICL TIGR gene indices clustering tools \\ Blast Basic Local Alignment Search Tool}

\section{Introduction}

Sugarcane belongs to the Poaceae family, which includes maize, wheat, rice, sorghum, and many types of grass $(\mathrm{Li}$ 2010). Cultivated sugarcane is an important industrial crop with a high sugar yield and has become a major energy crop worldwide (Grivet and Arruda 2001; Yang et al. 2010). It is cultivated in $\sim 26$ million hectares in tropical and subtropical regions of the world, producing up to 1.8 billion metric tons of crushable stems (Zhang et al. 2018). Sugarcane provides around $80 \%$ of the world's sugar, with the secondary production as raw materials for pulp, ethanol and bioplastics (Lam et al. 2009; Li and Yang 2015). Modern sugarcane cultivars are interspecific hybrids which were generated from the cross between Saccharum officinarum and Saccharum spontaneum, followed by backcrossing into Saccharum officinarum to select sugar-poor relative traits (Roah 1972; Branes and Sartoris 1936). In China, modern sugarcane is mainly distributed in the provinces of Guangxi, Yunnan, Guangdong, and Hainan. Some cultivars were also improved disease resistance by crossing with Saccharum barberi Jesweit or/and Narenga porphyrocoma (Gao et al. 2012; Liu et al. 2012a, b).

Sugarcane diseases, such as smut, white leaf, and wilt/top rot/Pokkah Boeng, are critical limitations of production, causing serious losses in yield and quality among susceptible cultivars (Hameed et al. 2015; Paulo et al. 2016; Su et al. 2016). Traditionally, the resistant traits of Saccharum spontaneum, Saccharum barberi Jesweit or/and Narenga porphyrocoma were introduced into Saccharum officinarum by hybridization to improve the disease resistance of cultivated sugarcane (Gao et al. 2012; Liu et al. 2012a, b). With the development of breeding approaches, molecular breeding with precise genome information accelerates the collection of disease resistance genes in one cultivar. However, applying this to sugarcane is difficult due to its high polyploidy and complex genome, with ploidy levels ranging from $5 \times$ to $16 \times$, and chromosome numbers from $2 \mathrm{n}=40-128$, with some even as high as 200 (Sreenivasan et al. 1987; Liu et al. 2012a, b). The estimated polyploid genome size of sugarcane ranges from 3.36 to $12.64 \mathrm{~Gb}$, and the monoploid genome size ranges from 760 to $985 \mathrm{Mb}$ (D'hont et al. 1996; Zhang et al. 2012), which is larger than the rice $(400 \mathrm{MB})$ and the sorghum $(760 \mathrm{MB})$ genomes (Soderlund et al. 2011). Such complex genetic background blocks the sequencing of the whole sugarcane genome. Studies on biological traits, such as biomass yield, sugar accumulation, and stress tolerance, have focused on transcriptome analysis (Kido et al. 2012; Fracasso et al. 2016; Huang et al. 2016). The transcriptome reveals specific transcripts produced under biotic stress (e.g., smut) and abiotic stress (e.g., drought) conditions in sugarcane (Iskandar et al. 2011; Mattielllo et al. 2015).

With the Saccharum spontaneum genome, which is relative high quality, as reference (Zhang et al. 2018), we employed transcriptome profiling to analyze the genes and pathways involved in twist leaf disease in modern sugarcane cultivar. Twisted leaf disease, caused by Phoma sp., which is one of the largest fungal genera, was first reported in Guangxi, China, in 2014, when more than 5\% of sugarcane was infected in the field. Twisted leaf disease is somewhat similar to Pokkah Boeng disease (caused by Fusarium moniliforme Sheldon). The symptoms begin with yellowing on the midribs and leaf margins, then spread to the entire leaf, along with twisting and curling of the crown leaves (Lin et al. 2014). The modern sugarcane cultivar used in this study was generated from the $\mathrm{BC}_{1}$ generation offspring of Narenga porphyrocoma via crossing and backcrossing with cultivated sugarcane varieties, so that it harbored the Narenga porphyrocom genetic background. The RNA-seq (two biological repeats) comparison between un-susceptible and susceptible cultivars in different stages of infection provided a reference for understanding the mechanism of twisted leaf disease. Analysis revealed that twisted leaf disease triggered the hormone network. $R$ genes expression profile showed $\sim 40 \% R$ genes expressed lower in susceptible cultivar. The null/retarded response of $R$ genes might be one of the possible reasons for $\mathrm{H} 3-8$ being susceptible to twisted leaf disease.

\section{Results}

\section{RNA Sequencing and Mapping to the Reference Genome}

Compared to the un-susceptible cultivar, the susceptible cultivar displayed twisted leaves after infection with the disease (Fig. 1a). To obtain the gene expression profile associated with twisted leaf disease in susceptible sugarcane, twelve sequencing cDNA libraries were constructed. They contained a susceptible genotype $\left(\mathrm{NSBC}_{1} \mathrm{~T}\right)$ and un-susceptible genotype $\left(\mathrm{NSBC}_{1} \mathrm{CK}\right)$ at three stages (pre-onset, early, and serious symptoms) (Fig. 1b) with two biological repeats (2 cultivars $\times 3$ stages $\times 2$ biological repeats). As shown in Table 1, a total of 258,301,460 and 279,952,436 raw reads were generated from the $\mathrm{NSBC}_{1} \_\mathrm{T}$ and $\mathrm{NSBC}_{1} \mathrm{CK}$ library by sequencing on the Illumina Hiseq 2000 platform, respectively. After filtering low-quality reads, unknown nucleotides, 
Fig. 1 The susceptible sugarcane clone H3-8 under twisted leaf disease stress. a Twisted leaf disease of $\mathrm{H} 3-8$ was observed in the field. b Phenotype of three stages of Phoma sp. infection. From top to bottom: pre-onset, early, and serious symptom stages a

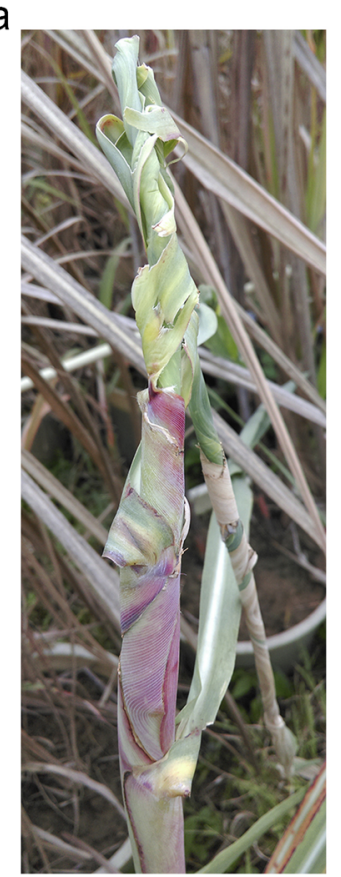

b

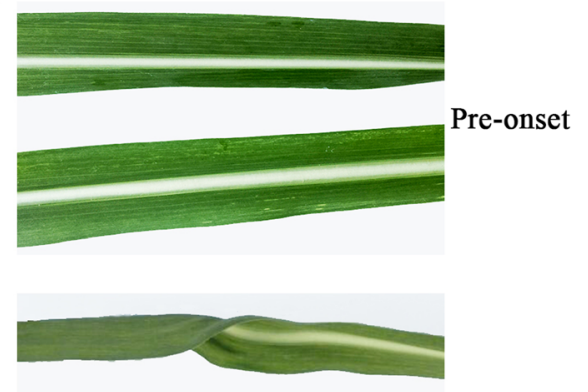

Early stage
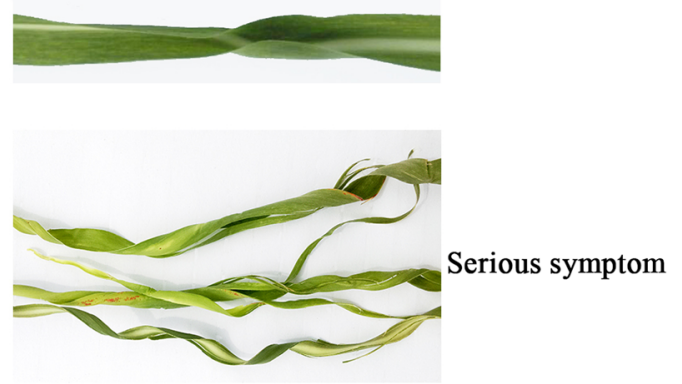

and the contained adapters, 251,482,172 and 273,757,964 clean reads were left in the $\mathrm{NSBC}_{1}$ T and $\mathrm{NSBC}_{1}$ CK libraries, respectively. Using the genome of Saccharum spontaneum as reference (Zhang et al. 2018), we mapped the clean reads to the reference via HISAT (hierarchical indexing for spliced alignment of transcripts) (Kim et al. 2015). All samples conditioned over $70 \%$ mapping ratio (average $76.74 \%$ ). The details were summarized in Table 2.

\section{Gene Expression Statistics}

To elucidate gene expression profile of un-susceptible and susceptible cultivars, we annotated the aligned reads according to the reference genome. The result showed that there were 91,386 genes expressed that included 65,391 known genes and 25,995 novel genes. In the 96,101 annotated transcripts, 53,481 transcripts with novel alternative splicing subtypes encode known proteins, 27,151 transcripts were defined as novel

Table 1 Overview of the transcriptome sequencing and de novo assembly results

\begin{tabular}{lll}
\hline Description & NSBC1_T & NSBC1_CK \\
\hline Raw reads & $258,301,460$ & $279,952,436$ \\
Q20 percentage & $55.56 \%$ & $55.61 \%$ \\
Clean reads & $251,482,172$ & $273,757,964$ \\
Total reads & $525,240,136$ & \\
Total nucleotides (nt) & $65,780,017,250$ & \\
\hline
\end{tabular}

protein coding genes, and 15,469 transcripts were classified into long non-coding RNAs. Details of each sample were exhibited in Table S1 and Fig. S1.

Based on the Pearson correlation coefficient of genes expression of each sample, we obtained the correlation heatmap of all samples (Fig. 2a). The samples from the same cultivar conditioned high correlation Pearson value. Of course, two biological repeats of each stage clustered together in the Cluster Dendrogram (Fig. 2b). That means two biological repeats of each stage were consistent and satisfied for further analysis.

Table 2 Genome mapping summary of all samples

\begin{tabular}{lll}
\hline Sample & Total Clean Reads & Total Mapping Ratio \\
\hline NSBC1_CK1-1 & $49,273,558$ & $76.43 \%$ \\
NSBC1_CK1-2 & $51,677,702$ & $77.54 \%$ \\
NSBC1_CK2-1 & $47,765,358$ & $71.71 \%$ \\
NSBC1_CK2-2 & $44,108,084$ & $75.97 \%$ \\
NSBC1_CK3-1 & $42,718,254$ & $77.39 \%$ \\
NSBC1_CK3-2 & $44,409,480$ & $76.30 \%$ \\
NSBC1_T1-1 & $44,375,910$ & $77.42 \%$ \\
NSBC1_T1-2 & $42,446,806$ & $78.30 \%$ \\
NSBC1_T2-1 & $23,302,262$ & $78.14 \%$ \\
NSBC1_T2-2 & $23,302,264$ & $77.40 \%$ \\
NSBC1_T3-1 & $42,487,034$ & $76.63 \%$ \\
NSBC1_T3-2 & $41,604,074$ & $77.65 \%$ \\
\hline
\end{tabular}


Fig. 2 Correlation analysis of all samples. a Heatmap was constructed by the Pearson correlation coefficient of genes expression. b Cluster analysis of all samples based on genes expression quantity a

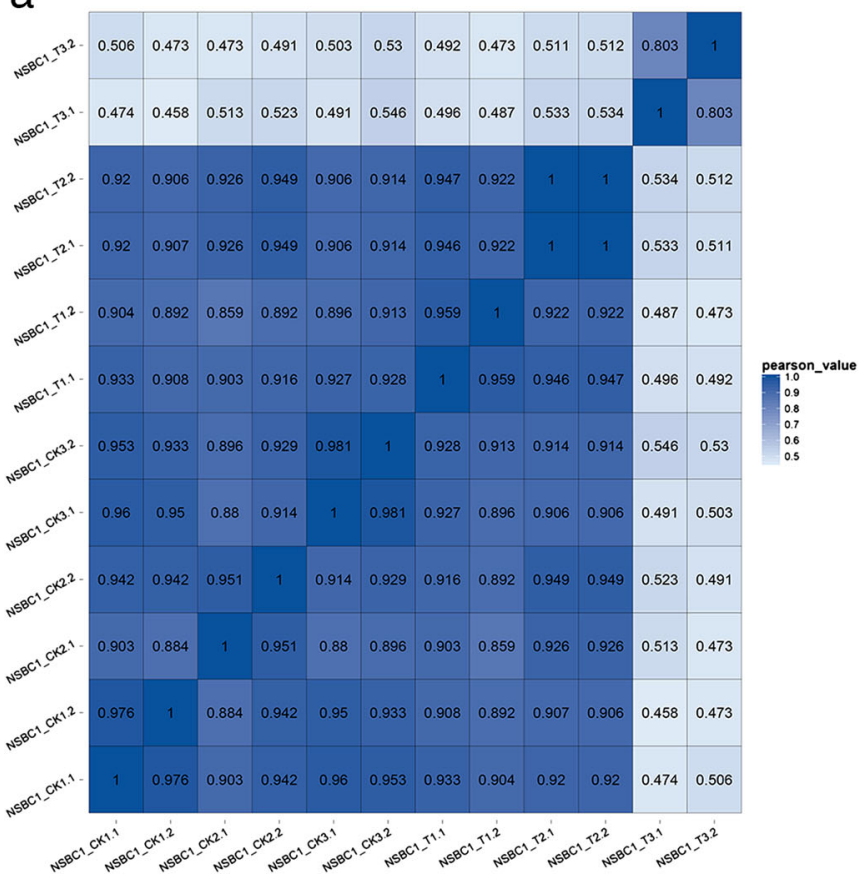

$\mathrm{b}$

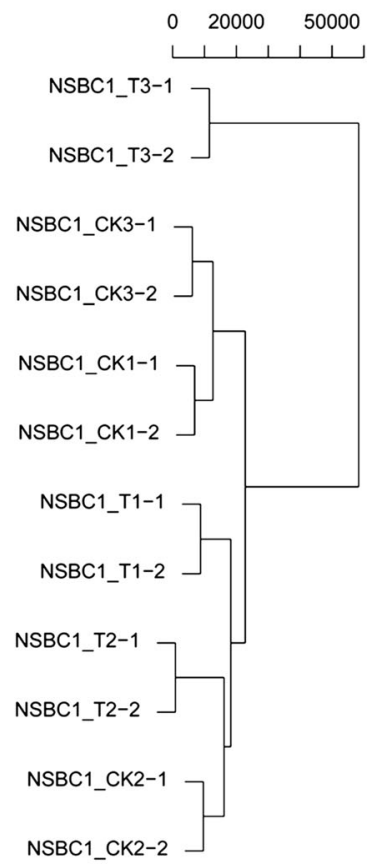

\section{Differentially Expressed Genes (DEGs) in Un-Susceptible and Susceptible Cultivars}

The significance of the gene expression difference was determined according to the threshold of FDR (False Discovery Rate) $<0.05$ and $\mid \log _{2}$ FC (Fold Change) $\mid>1$. In the comparison to the un-susceptible clone $\mathrm{H} 3-19$, the genes expression of susceptible clone H3-8 showed 8408 up-regulated and 7834 down-regulated in pre-onset stage, 7334 up-regulated and 5558 down-regulated in early stage, and 24,456 upregulated and 10,468 down-regulated in serious symptom stage. (Fig. 3). The Gene Ontology (GO) analysis classified DEGs into three main categories: biological function, cellular component, and molecular function (Fig. 4). DEGs mainly accumulated in the cellular process, metabolic process, and biological regulation in the biological function. The cell, cell part, and organelle conditioned most enriched DEGs in the cellular component. In the term of molecular function, DEGs were mainly distributed in catalytic activity and binding. Most enriched GO term are corresponding to various aspects of activated metabolism (e.g. "metabolic process" and "catalytic activity") and stress response (e.g. "biological regulation" and "organelle"). We also performed a BLAST (The Basic Local Alignment Search Tool) (E-value $<0.00001$ ) analysis of the DEGs against the KEGG (Kyoto encyclopedia of genes and genomes) database. The DEGs were mainly enriched in metabolic pathways, biosynthesis of secondary metabolites, and plant-pathogen interaction (Fig. S2). These are consistent with the sugarcane activities in response to Phoma sp. infection. According to these findings, we analyzed specific pathways and genes related to disease defense.
Fig. 3 DEG analysis in the susceptible cultivars. Numbers of up- and down-regulated genes identified in the pre-onset, early, and serious symptom stages in susceptible genotypes

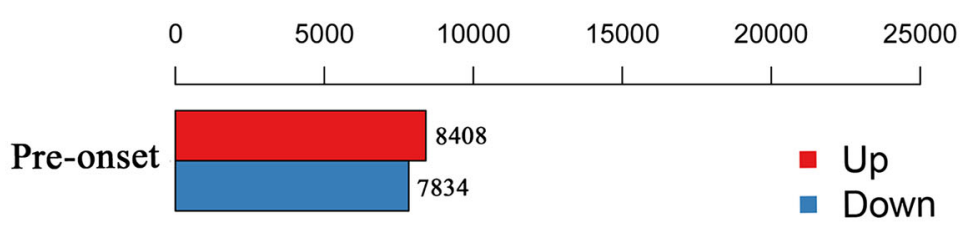

\begin{tabular}{l|l|l}
\hline Early stage & \multicolumn{2}{|c}{7334} \\
& 5558
\end{tabular}

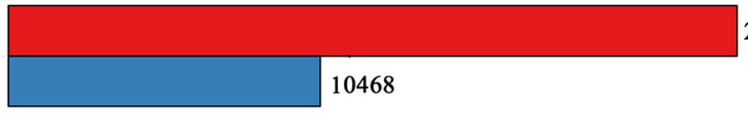




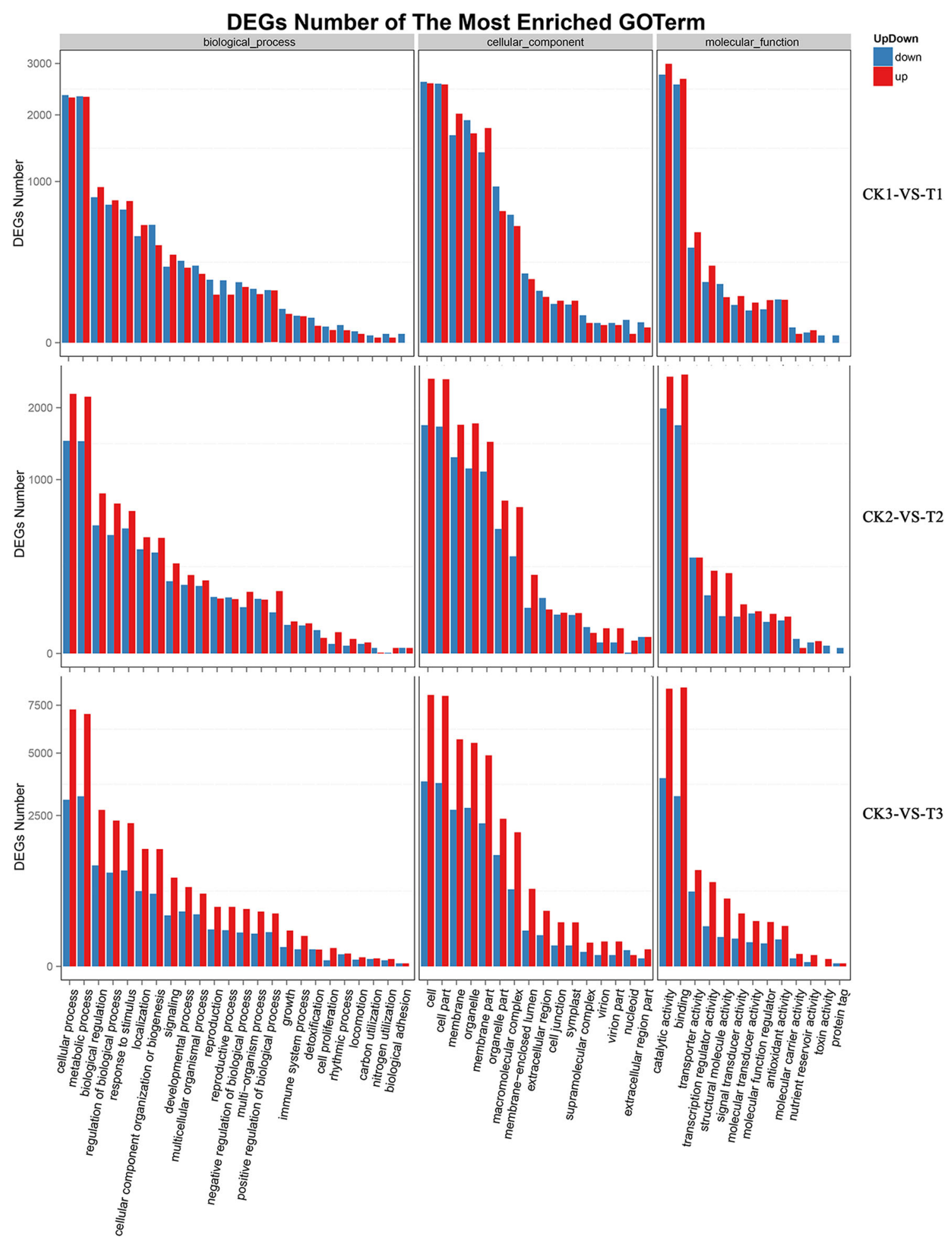

Fig. 4 Gene ontology (GO) annotation for DEGs in three stages

\section{Ingenuity Pathways Analysis}

Plant hormones are the first reactors under environmental stress (Verma et al. 2016). Among the major hormones, abscisic acid (ABA), ethylene (ET), jasmonates (JA), and salicylic acid (SA) play essential roles in regulating plant response to biotic and abiotic stress (Bari and Jones 2009). The DEGs between the susceptible and un-susceptible cultivars provided us an opportunity to study how the hormone pathways response to twisted leaf disease. We analyzed the expression patterns of genes involved in hormone biogenesis in three different stage of Phoma sp. infection (Fig. 5; 
Fig. 5 Ingenuity analysis of DEGs in hormone pathways during fungi infection process. Fold change $\left(\log _{2}\right)$ in hormone biosynthesis genes at different stages of the infection process relative to the resistance condition

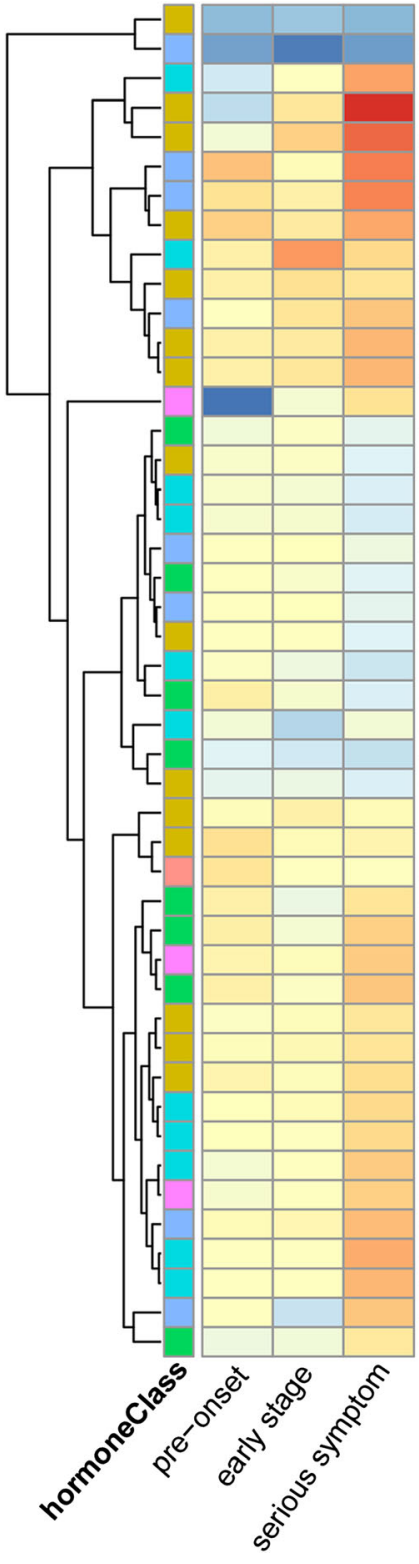

Sspon.01G0004390-4D Sspon.03G0015850-3C LOC_Os05g39320-D26 LOC-Os03g49380-D82 Sspon.01G0049070-1B Sspon.05G0004850-3D Sspon.07G0013040-1A Sspon.08G0011550-4D Sspon.02G0020760-1A Sspon.04G0019150-2D LOC Os04g48850-D4 Sspon.01G0025830-1A Sspon.01G0046850-2C LOC Os03g44380-D6 Sspon.02G0052050-1C Sspon.02G0032580-1A LOC Os01g56380-D7 LOC_Os06g23140-D3 Sspon.08G0016450-2D Sspon.06G0005400-4D LOC_Os06g03990-D2 Sspon.08G0010980-1A LOC_Os01g12770-D245 Sspon.04G0008040-6P Sspon.02G0033930-1B Sspon.02G0016020-4D Sspon.03G0009710-3D LOC Os02g10120-D1 LOC_Os06g11290-D5 Sspon.01G0031030-1A Sspon.04G0008070-3C LOC Os02g41630-D10 Sspon.02G0051940-1C Sspon.05G0007010-4D Sspon.03G0012630-1A Sspon.07G0000210-2B Sspon.06G0001670-1A LOC Os03g57690-D17 Sspon. 02G0044400-2D LOC_Os12g32750-D2 Sspon.01G0024170-2D Sspon.07G0013030-3C Sspon.01G0030000-1A Sspon.07G0005790-1A Sspon.03G0022900-1A Sspon.04G0017080-3P
10 hormoneClass

abscisic acid biosynthesis

5 auxin biosynthesis cytokinin biosynthesis ethylene biosynthesis

$0 \quad$ jasmonic acid biosynthesis salicylic acid biosynthesis

$-5$

$-10$

Table S1). As the main response hormones in biotic stress, the biosynthesis of SA, JA, and ET up-regulated in the susceptible cultivar (genes in green, brown, and blue lines). The SA, which is generally involved in the defense against biotrophic and hemi-biotrophic pathogens (Loake and Grant 2007), was enriched with high expression ( $\sim 4$ folds up-regulated) of LOC_Os02g41630-D10and Sspon.04G0008070-3C (Fig. 5, green lines). JA and ET, which involve in the defense of necrotrophic pathogens and herbivorous insects (Gamalero and Glick 2012; Wasternack and Hause 2013), accumulated through up-regulating biosynthesis genes (Fig. 5, brown lines). ABA, auxin (IAA), and cytokinins (CK) biosynthesis genes also exhibited differential expressions in the susceptible cultivar, which resulted from hormone pathways crosstalk and secondary plant reflections (Robert-Seilaniantz et al. 2011). These results show that twisted leaf disease triggered the reaction of the hormones network.

Also, we analyzed the specific biosynthesis genes in SA, JA and ET pathway, respectively. In details, the phenylalanine ammonia-lyase, an enzyme of phenylalanine ammonium lyase (PAL) pathway in SA synthesis (Yokoo et al. 2018), was increased in expression at the pre-onset of Phoma sp. infection. The isochorismate synthase 1 , which is involved in the synthesis of SA (Yokoo et al. 2018), showed lower expression level in three stages of the susceptible cultivar (Table S1). In the JA pathway, 12-oxophytodienoate reductase, which catalyses the reduction of 10,11-double bonds of 12oxophytodienoate to 3-oxo-2-(2'-pentenyl)-cyclopentane-1- 
octanoic acid (OPC-8:0) (Tani et al. 2008), conditioned relative higher expression in the susceptible cultivar (Table S1). The aminotransferase, a gene family including VAS1 which participates in auxin and ethylene biosynthesis (Zhang et al. 2012), varied in expression at different stages of the twisted leaf disease. These findings supported that the infection of Phoma sp. induced the response of the hormones network.

\section{Evaluation of Differentially Expressed Disease-Resistant Genes}

To defend against infection of pathogen, the expression of resistance $(R)$ genes that encode proteins containing conserved nucleotide binding site plus leucine-rich repeat (NBSLRR domain) are induced (Bakker et al. 2006). To elucidate the $R$ genes involved in the twisted leaf disease, we annotated $R$ genes in DEGs based on PRG (Pathogen receptor genes) database. (Table 3). There were $46 \%, 42 \%$, and $26 \% R$ genes conditioned lower expression in pre-onset, early stage, and serious symptom of susceptible cultivar relative to the control, respectively. That means a part of $R$ genes in the susceptible cultivar were null/retarded in the response of Phoma sp. infection.

From the $R$ genes expression profile, we selected out representative of each class to exhibit their expression patterns in three stages of Phoma sp. infection (Fig. 6). Sspon.06G0034670-1D, Sspon.04G0004450-1A and Sspon.02G0029950-1T dramatically decreased in three stages of Phoma sp. infection. Sspon.01G0011870-1A and Sspon.01G0013680-3C also showed lower expression level in the three stages. $R$ genes like these genes were null function in the susceptible cultivar. Sspon.05G0016050-1A, Sspon.08G0026530-1C and Sspon.03G0025750-3D expressed lower at pre-onset and kept increasing in the later stages. That means $R$ genes with such expression patterns in the susceptible cultivar were retarded in the defense of Phoma sp. infection. Some $R$ genes like Sspon.02G0020920-2B, Sspon.02G0041050-1B, and Sspon.01G0037110-1B, conditioned higher expression or/and irregular expression patterns in three stages. These $R$ genes could respond to Phoma sp. infection in the susceptible cultivar. Also, the sequence analysis of these representative genes, including alternative splicing and SNPs, didn't offer clue for the structural mutation in the susceptible cultivar (data not shown). The expression level analysis of $R$ genes revealed that the null and retarded response of $R$ genes in the Phoma sp. infection, especially at the pre-onset stage might be one of the reasons that the H3-8 cultivar is susceptible to twisted leaf disease.

\section{Discussion}

The transcriptome, which is influenced by external environmental conditions, can effectively reveal the response mechanism of biotic and abiotic stress in plants (Wei et al. 2011). In our study, the natural infected cultivar H3-8 was defined as susceptible cultivar and the un-susceptible cultivar (H3-19) was used as control. With two biological repeats, we used transcriptome profiling to detect the genes associated with twisted leaf disease and obtained 65,780,017,250 bp data from three stages of the twisted leaf disease. Using the genome of Saccharum spontaneum L. as reference (Zhang et al. 2018), the average mapping ratio of twelve samples was $76.74 \%$. The annotation of aligned reads showed 91,386 genes expressed (65,391 known and 25,995 novel), and 96,101 annotated transcripts, including 53,481 novel alternative splicing subtypes
Table 3 Classification of annotated $R$ genes and representatives for ingenuity analysis

\begin{tabular}{|c|c|c|c|}
\hline Class & Count & Representatives & Description \\
\hline NL & 543 & Sspon.02G0020920-2B & Disease resistance protein (NBS-LRR class) family \\
\hline RLP & 683 & Sspon.02G0041050-1B & $\begin{array}{l}\text { Pyridoxal phosphate-dependent transferases superfamily } \\
\text { protein }\end{array}$ \\
\hline CNL & 242 & Sspon.05G0016050-1A & HOPZ-ACTIVATED RESISTANCE 1 \\
\hline $\mathrm{N}$ & 309 & Sspon.08G0026530-1C & NB-ARC domain-containing disease resistance protein \\
\hline TNL & 100 & Sspon.06G0034670-1D & $\begin{array}{l}\text { TIR-NBS-TIR-TIR-WRKY type disease resistance } \\
\text { protein }\end{array}$ \\
\hline RLK & 7 & BGI_novel_G009740 & S-locus lectin protein kinase family protein \\
\hline RLK-GNK2 & 94 & Sspon.01G0015790-1A & cysteine-rich RLK (RECEPTOR-like protein kinase) \\
\hline $\mathrm{CN}$ & 67 & Sspon.04G0004450-1A & $\begin{array}{l}\text { LRR and NB-ARC domains-containing disease resistance } \\
\text { protein }\end{array}$ \\
\hline Other & 40 & Sspon.01G0037110-1B & NADPH HC toxin reductase-like protein \\
\hline $\mathrm{T}$ & 17 & Sspon.01G0011870-1A & NAC domain containing protein \\
\hline Mlo-like & 21 & Sspon.01G0013680-3C & Seven transmembrane MLO family protein \\
\hline $\mathrm{L}$ & 5 & Sspon.03G0025750-3D & Protein phosphatase 1 regulatory subunit \\
\hline RPW8-NL & 4 & Sspon.02G0029950-1T & ADR1-like 1 disease resistance protein \\
\hline
\end{tabular}




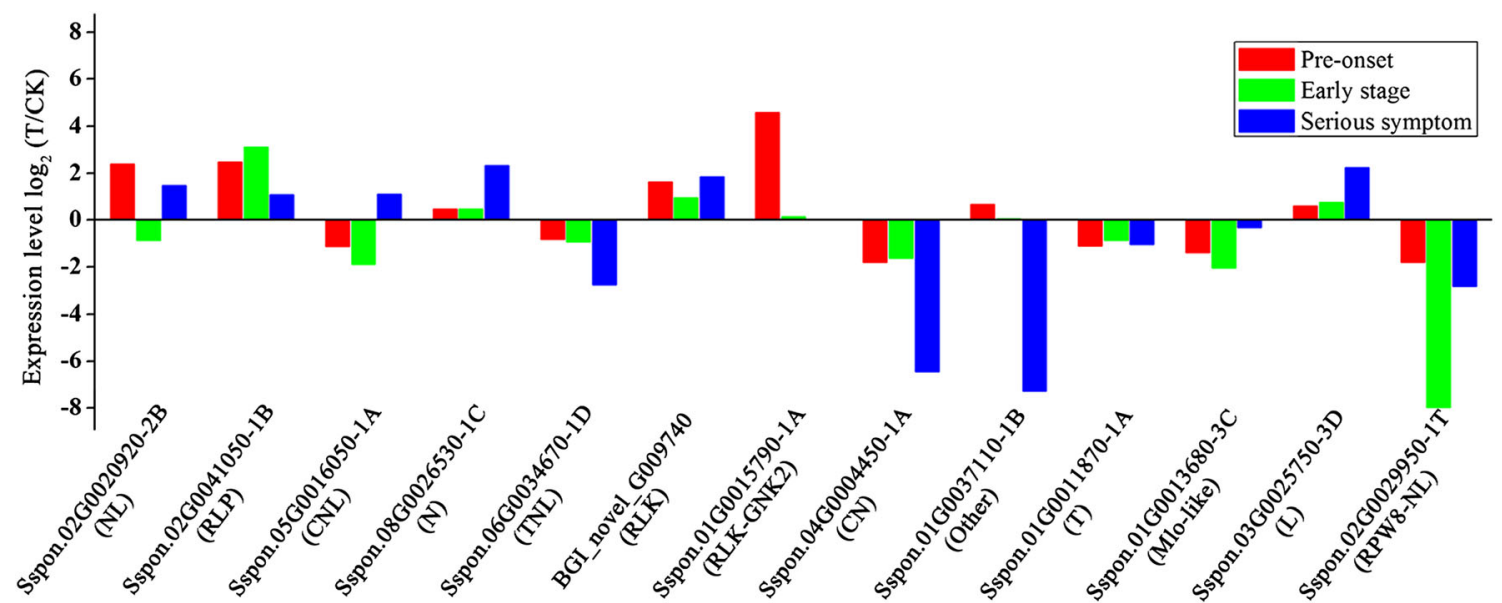

Fig. 6 Differential expression analysis of specific $R$ genes in the susceptible cultivar. Genes were selected out as representative based the tendency of each $R$ gene class. Fold change $\left(\log _{2}\right)$ was achieved from the FPKM value of T and CK

encode known proteins, 27,151 novel protein coding genes, and 15,469 long non-coding RNAs. With these data, we analyzed the DEGs, including hormone pathways and $\mathrm{R}$ genes, between the susceptible cultivar and the control. Also, these data provided a foundation for the further analysis of the twisted leaf disease, such as specific gene characterization, resistant genes selection, and immune network description.

The comparison between susceptible cultivar and the control identified 16,242, 12,892 and 34,924 DEGs in pre-onset, early stage and serious symptom stage, respectively (Fig. 3). These DEGs are involved in critical biological activities that are essential for disease resistance (Fig. 4). Ingenuity pathway analysis revealed hormone biosynthesis genes variations in the susceptible cultivar (Fig. 5). The SA and ET pathways were induced in the infection of Phoma sp. at the pre-onset stage. Along with the spread of the disease, JA and ET synthesis increased and maintained high levels in the serious symptom stages. As three major hormones involved in stress resistance, SA is a biotrophic and hemi-biotrophic pathogentriggered signaling pathway, and JA combined with ET signaling pathways are induced by necrotrophic pathogens (Glazebrook 2005). Therefore, twisted leaf disease, caused by fungal pathogens, first triggers the SA pathway as response to pathogens infection. The high level of JA and ET in serious symptoms might partially result from tissue damage in disease. Other hormones, including ABA, auxin, and $\mathrm{CK}$, crosstalk with major disease response hormones (SA, JA, and ET) and construct a network to respond to disease stress (Verma et al. 2016).

Plant disease resistance $(R)$ genes are prevalent in all plant species and harbor a conserved LRR domain (Meyers et al. 1998). $R$ genes are defined as gene-for-gene interaction and specifically recognize an avirulence protein encoded by a pathogen with a hypersensitive response (Flor 1956). In the $R$ gene analysis of the susceptible cultivar, around $40 \% R$ genes, including those that play essential functions, were down-regulated, especially in pre-onset stage. For example, belongs to LRR and NB-ARC domains-containing proteins of which members participate in disease resistance (Fischer et al. 2016). is predicted to encode RGA protein which contains NB-ARC domain and functions disease resistance (Cesari et al. 2013). is descripted as CRKs (cysteine-rich protein kinase), which involves in the ROS signaling pathway and plays important roles in the elimination of fungal growth damage (Niina Idänheimo 2015). The low expression of these $R$ genes in the twisted leaf disease would drag down the rapid response of $R$ genes defensive system. Besides $\sim 40 \%$ downregulated $R$ genes, there were still a number of $R$ genes conditioned normal expression level or even higher expression level in the susceptible cultivar. Unfortunately, these high expressed $R$ genes were unable to eliminate the pathogens successfully. These increased expressions might be a result of universal reactions of sugarcane under pathogens stress rather than the specific response of Phoma sp. infection. Such phenomenon was commonly reported in the studies of other species (Heath 2000; van Loon 2015).

\section{Materials and Methods}

\section{Plant Materials and Sampling}

Narenga porphyrocoma, which is an important relative genus of sugarcane, was collected from a barren mountainous area in Guangxi province, China. We obtained the $\mathrm{BC}_{1}$ generation offspring of the Narenga porphyrocoma via crossing and backcrossing with sugarcane varieties several years. Among the $\mathrm{BC}_{1}$ offspring, $\mathrm{H} 3-8$ was susceptible to twisted leaf disease at the sugarcane elongating stage, whereas H3-19 was not. H3-8 was proven to affect stable twisted leaf disease occurrence after 3 years based on field and greenhouse observations. 
Plantlets at the same growth stage were selected and planted in pots filled with a mixture of peat soil and washed sand, and grown in a greenhouse at the Sugarcane Research Institute, Guangxi Academy of Agricultural Sciences. The natural infected plants were selected and defined as the susceptible cultivator. The leaf samples of the susceptible sugarcane clone $\mathrm{H} 3-8$ were collected at three stages corresponding to the pre-onset (named $\mathrm{NSBC}_{1} \mathrm{~T} 1$ ), early (named $\mathrm{NSBC}_{1} \_\mathrm{T} 2$ ), and serious symptom (named $\mathrm{NSBC}_{1} \_\mathrm{T} 3$ ) stages of the disease, respectively. The leaf samples of the un-susceptible sugarcane clone $\mathrm{H} 3-19$ were simultaneously collected, labeled $\mathrm{NSBC}_{1} \mathrm{CK} 1, \mathrm{NSBC}_{1} \mathrm{CK} 2$, and $\mathrm{NSBC}_{1} \mathrm{CK}_{3}$, respectively. Two biological repeats of each sample were collected. All samples were immediately frozen in liquid nitrogen and stored at $-80^{\circ} \mathrm{C}$.

\section{RNA Extraction and Quality Determination}

The total RNA was extracted using TRIzol reagent (Invitrogen, Carlsbad, CA, USA) and treated with RNasefree DNase I and RNA integrity number (RIN > 8.0). RNA quality and quantity were verified using a NanoDrop 1000 spectrophotometer (Wilmington, DE, CA, USA) and an Agilent 2100 Bioanalyzer (Santa Clara, CA, USA) prior to the library construction. No sign of degradation was found.

\section{CDNA Library Construction and Sequencing}

The poly(A) RNA was fragmented into approximately 300-nt fragments using RNA Fragmentation Reagents (Ambion, Austin, TX, USA). Using these short fragments as templates, the double-stranded cDNA was synthesized with random primers (Invitrogen, Carlsbad, CA, USA). The end repair of these cDNA fragments was subsequently performed with Klenow polymerase, T4 DNA polymerase, and T4 polynucleotide kinase (NEB,Ipswich, MA, USA). Illumina adapters (containing primer sites for sequencing and flow cell surface annealing) were ligated to the end-repaired fragments using T4 DNA ligase (Invitrogen, Carlsbad, CA, USA). The products were enriched for the cDNA fragments using a Qiaquick Gel Extraction Kit (Qiagen, Duesseldorf, Germany) and amplified with polymerase chain reaction (PCR) to prepare the sequencing library. Agilent 2100 Bioanalyzer (Agilent, Beijing, China) was used to detect the quantity and quality of the cDNA. Then, the paired-end RNA-seq libraries were prepared following Illumina's protocols and sequenced on the Illumina HiSeq 2500 platform (Illumina, San Diego, CA, USA). Sequencing was performed in a flowcell on an Illumina HiSeq 2500 sequencer using the TruSeq PairedEnd Cluster Kit v3 (Illumina PE-401-3001) and the TruSeq SBS HS Kit v3200 cycles (Illumina FC-401-3001), generating $2 \times 125 \mathrm{bp}$.

\section{Data Filtering and Genome Mapping}

The raw reads were filtered according to the following criteria: (1) reads containing adaptor, (2) reads with unknown nucleotides larger than $5 \%$, and (3) low-quality reads (the rate of reads with a quality value of $\leq 10$ was more than $20 \%$. The clean data was mapped to the genome of Saccharum spontaneum L. (Zhang et al. 2018) via HISAT (v2.0.4; -phred64 -sensitive -no-discordant -no-mixed -I 1 -X 1000) (Kim et al. 2015). The transcripts were re-constructed via StringTie and predicted using Cuffcompare tool in Cufflink (Pertea et al. 2003; Trapnell et al. 2010; Pertea et al. 2016).

\section{Differential Gene Expression}

The DEGs analysis was performed as the description of DEGseq (Wang et al. 2010) and corrected the $P$-values to $Q$-values based on Benjamini-Hochberg method (Benjamini and Hochberg 1995) and Storey-Tibshirani method (Storey and Tibshirani 2003). GO (gene ontology) terms were assigned based on the best-hits BLASTx resulted from NR alignments that were derived from Blast2GO (v2.5.0) against GO database (release20,120,801). The DEGs were aligned against KEGG (Kyoto Encyclopedia of Genes and Genomes) by BLASTx package with threshold of E-value $<=10-5$.

\section{Ingenuity Analysis of DEGs}

The rice hormones proteins were used as reference to identify the hormone genes in sugarcane (Cohen et al. 2017). The longest transcript of genes was selected as representative for analysis. All predicted protein sequences were aligned against the sugarcane gene models by BLASTp (Identity $>50 \%$; Coverage $>50 \%$; E-value: $\left.1 \times 10^{7}\right)$, and defined as homologs of hormones biosynthesis genes. Also, we aligned all predicted hormone genes against the sugarcane genome via tBLASTn (Evalue: 1E-10), and used GeneWise (v2.2.0) to identify the structure of candidate hormone genes. Among these genes, the ones with non-significant different expression in three stages were eliminated. The final candidates were aligned against KEGG database (version:84). The $\log$ fold of each gene was obtained from the casecontrol group. The patterns of the gene in each group were compared. Similarly, predicted proteins were aligned against the PRG database using BLASTp. The results were filtered (E-value: 1e10), and the best hit of predicted protein was retained. The fpkm (Reads per kilobase of exon per million reads mapped) of each predicted resistant gene in the case sample was filtered out and a downstream analysis was performed. 
Acknowledgments We thank Mick Rose in Primary Industry Department of Australia for the assistance of writing paper.

Author Contributions Hongwei Tan, and Xihui Liu conceived and designed the experiments. Jinju Wei and Huiping Ou performed the experiments. Xiaoqiu Zhang, Ronghua Zhang, Hui Zhou, Yiyun Gui,Haibi Li, Yangrui Li, Rongzhong Yang and Dongliang Huang performed cross and backcross of Narenga porphyrocoma, planted sugarcane and collected samples. Zhihui Xiu, Junhui Chen and Huayan Jiang analyzed the data. Zhihui Xiu and Xihui Liu wrote the manuscript. All authors have read and approved the final manuscript.

Funding This work was financially supported by the National Science Foundation of China (31760368, 31101195), Guangxi Fund (GKAA17202042-6), Fund of Guangxi Academy of Agricultural Sciences (GNK2018YT02 and GNK2018YM01) and Fund of Modern Agriculture Technology (CARS-170105, gjnytxgxcxtd-03).

\section{Compliance with Ethical Standards}

Conflict of Interest The authors declare no conflict of interest.

The data that support the findings of this study have been deposited in the CNSA (https://db.cngb.org/cnsa/) of CNGBdb with accession code CNP0000220, and NCBI with accession code SAMN05428728 to SAMN05428739.

Open Access This article is distributed under the terms of the Creative Commons Attribution 4.0 International License (http:// creativecommons.org/licenses/by/4.0/), which permits unrestricted use, distribution, and reproduction in any medium, provided you give appropriate credit to the original author(s) and the source, provide a link to the Creative Commons license, and indicate if changes were made.

\section{References}

Bakker EG, Toomajian C, Kreitman M, Bergelson J (2006) A genomewide survey of $\mathrm{R}$ gene polymorphisms in Arabidopsis. Plant Cell 18(8):1803-1818

Bari R, Jones JD (2009) Role of plant hormones in plant defence responses. Plant Mol Boil 69:473-488

Benjamini Y, Hochberg Y (1995) Controlling the false discovery rate: a practical and powerful approach to multiple testing. J R Stat Soc Ser B 57:289-300

Branes EW, Sartoris GB (1936) Sugarcane: its origin and improvement. Yerab US Dep Agric:561-624

Cesari S, Thilliez G, Ribot C, Chalvon V, Michel C, Jauneau A, Rivas S, Alaux L, Kanzaki H, Okuyama Y, Morel JB, Fournier E, Tharreau D, Terauchi R, Kroj T (2013) The rice resistance protein pair RGA4/RGA5 recognizes the Magnaporthe oryzae effectors AVRpia and AVR1-CO39 by direct binding. Plant Cell 25(4):1463-1481

Cohen SP, Liu H, Argueso CT, Pereira A, Vera Cruz C, Verdier V, Leach JE (2017) RNA-Seq analysis reveals insight into enhanced rice Xa7mediated bacterial blight resistance at high temperature. PLoS One 12(11):e0187625

D’Hont A, Grivet L, Feldmann PRS, Berding N, Glaszmann JC (1996) Characterisation of the double genome structure of modern sugarcane cultivars (Saccharum spp.) by molecular cytogenetics. Mol Gen Genet 250:405-413

Fischer I, Dievart A, Droc G, Dufayard JF, Chantret N (2016) Evolutionary dynamics of the leucine-rich repeat receptor-like kinase (LRR-RLK) subfamily in angiosperms. Plant Physiol 170: $1595-1610$

Flor HH (1956) The complementary genic system in flax and flax rust. Adv Genet 8:29-54

Fracasso A, Trindade LM, Amaducci S (2016) Drought stress tolerance strategies revealed by RNA-Seq in two sorghum genotypes with contrasting WUE. BMC Biol 16:115

Gamalero E, Glick BR (2012) Ethylene and abiotic stress tolerance in plants. In: Environmental adaptations and stress tolerance of plants in the era of climate change. Springer, New York, pp 395-412

Gao Y, Fang F, Liu XH et al (2012) Identification of progeny from crosses between sugarcane (Saccharum spp.) and intergeneric hybrid complex (Erianthus arundinaceus $\times$ Saccharum spontaneum) with molecular markers. J Plant Genet Res 13:912-916

Glazebrook J (2005) Contrasting mechanisms of defense against biotrophic and necrotrophic pathogens. Annu Rev Phytopathol 43: 205-227

Grivet L, Arruda P (2001) Sugarcane genomics: depicting the complex genome of an important tropical crop. Curr Opin Plant Biol 5:122-127

Hameed U, Pan YB, Iqbal J (2015) Genetic analysis of resistance gene analogues from a sugarcane cultivar resistant to red rot disease. J Phytopathol 163:755-763

Heath MC (2000) Nonhost resistance and nonspecific plant defenses. Curr Opin Plant Biol 3:315-319

Huang DL, Gao YJ, Gui YY, Chen ZL, Qin CX et al (2016) Transcriptome of high sucrose sugarcane variety GT35. Sugar Tech 18:520-528

Idänheimo N (2015) The role of cysteine-rich receptor-like protein kinases in ROS signaling in Arabidopsis thaliana. Department of Biosciences, University of Helsinki, Finland

Iskandar HM, Casu RE, Fletcher AT, Schmidt S, Xu JS et al (2011) Identification of drought-response genes and a study of their expression during sucrose accumulation and water deficit in sugarcane culms. BMC Plant Bio 11:12

Kido EA, Ferreira NJRC, de Oliveira Silva RL, Pandolfi V, Guinaraes ACR et al (2012) New insights in the sugarcane transcriptome responding to drought stress as revealed by supersage. Scientific World J 2012:821062

Kim D, Langmead B, Salzberg SL (2015) HISAT: a fast spliced aligner with low memory requirements. Nat Methods 12:357-360

Lam E, Shine J, Da SJ, Lawton M, Bonos S et al (2009) Improving sugarcane for biofuel: engineering for an even better feedstock. GCB Bioenergy 1:251-255

Li YR (2010) Modern sugarcane science. Beijing: China Agriculture Press 1-125

Li YR, Yang LT (2015) Research and development priorities for sugarcane industry of China: resent research hignlights. Sugar Tech 17:912

Lin ZY, Que YX, Deng ZH, Xu SQ, Rao GP et al (2014) First report of Phoma sp. caused twisting and curling of crown leaves of sugarcane in mainland of China. Plant Dis 98:850

Liu XH, Fang FX, Gao YJ, Zhang RH, Song HZ et al (2012a) Identification and genetic analysis of hybrid from cross between Erianthus arundinacius (Retz.) Jesws. And Saccharum spontaneum L. Acta Agron Sin 38:914-920

Liu XH, Fang FX, Zhang RH, Song HZ, Gao YJ et al (2012b) Identification of progenies from sugarcane $\times$ Narenga porphyrocoma (Hance) Bor. by SSR marker. Southwest China J Agric Sci 25:38-43

Loake G, Grant M (2007) Salicylic acid in plant defence-the players and protagonists. Curr Opin Plant Biol 10:466-472

Mattiello L, Riaño-Pachón DM, Martins MCM, da Cruz LP, Bassi D et al (2015) Physiological and transcriptional analyses of developmental stages along sugarcane leaf. BMC Plant Bio 15:300 
Meyers BC, Shen KA, Rohani P, Gaut BS, Michelmore RW (1998) Receptor-like genes in the major resistance locus of lettuce are subject to divergent selection. Plant Cell 10:1833-1846

Paulo C, Sentelhas DL, dos Santos Leonardo A et al (2016) Agro-climatic favorability zones for sugarcane orange rust as a tool for cultivar choice and disease management. Crop Prot 84:88-97

Pertea G, Huang XQ, Liang F, Antonescu V, Sultana R, Karamycheva S, Lee Y, White J, Cheung F, Parvizi B, Tsai J, Quackenbush J (2003) TIGR gene indices clustering tools (TGICL): a software system for fast clustering of large EST datasets. Bioinformatics. 19:651-652

Pertea M, Kim D, Pertea GM, Leek JT, Salzberg SL (2016) Transcriptlevel expression analysis of RNA-seq experiments with HISAT, StringTie and Ballgown. Nat Protoc 11:1650-1667

Roah BT (1972) Nobilization of sugarcane. Proc Int Soc Sugar Cane Technol 14:206-216

Robert-Seilaniantz A, Grant M, Jones JD (2011) Hormone crosstalk in plant disease and defense: more than just jasmonate-salicylate antagonism. Annu Rev Phytopathol 49:317-343

Soderlund C, Bomhoff M, Nelson WM (2011) SyMAP v3.4: a turnkey synteny system with application to plant genomes. Nucl Acids Res 2011:1-9

Sreenivasan TV, Ahloowalia BS, Heinz DJ (1987) Cytogenetics: in: Heinz DJ (ed) sugarcane improvement through breeding. Elsevier, New York, pp 211-253 Cytogenetics

Storey JD, Tibshirani R (2003) Statistical significance for genomewide studies. Proc Natl Acad Sci U S A 100:9440-9445

Su YC, Guo JL, Ling H, Chen SS, Wang SS et al (2016) Isolation of a novel peroxisomal catalase gene from sugarcane, which is esponsive to biotic and abiotic stresses. PLoS One 9(1):e84426

Tani T, Sobajima H, Okada K, Chujo T, Arimura S, Tsutsumi N, Nishimura M, Seto H, Nojiri H and Yamane H (2008) Identification of the OsOPR7 gene encoding 12-oxophytodienoate reductase involved in the biosynthesis of jasmonic acid in rice. Planta 227:517-526

Trapnell C, Williams BA, Pertea G, Mortazavi AM, Kwan G, van Baren MJ, Salzberg SL, Wold B, Pachter L (2010) Transcript assembly and quantification by RNA-Seq reveals unannotated transcripts and isoform switching during cell differentiation. Nat Biotechnol 28:511-515

van Loon LC (2015) The intelligent behavior of plants. Trends Plant Sci 21:286-294

Verma V, Ravindran P, Kumar PP (2016) Plant hormone-mediated regulation of stress responses. BMC Plant Boi 16:86

Wang L, Feng Z, Wang X, Wang X, Zhang X (2010) DEGseq: an R package for identifying differentially expressed genes from RNAseq data. Bioinformatics. 26:136-138

Wasternack C, Hause B (2013) Jasmonates: biosynthesis, perception, signal transduction and action in plant stress response, growth and development. An update to the 2007 review in annals of botany. Ann Bot 111(6):1021-1058

Wei W, Qi X, Wang L, Zhang Y, Hua W, Li D, Lv H, Zhang X (2011) Characterization of the sesame (Sesamum indicum L.) global transcriptome using Illumina paired-end sequencing and development of EST-SSR markers. BMC Genomics 12:451

Yang S, Vanderbeld B, Wan J, Huang Y (2010) Narrowing down the targets: towards successful genetic engineering of drought-tolerant crops. Mol Plant 3:469-490

Yokoo S, Inoue S, Suzuke N, Amakawa N et al (2018) Comparative analysis of plant isochorismate synthases reveals structural mechanisms underlying their distinct biochemical properties. Biosci Rep 38:BSR20171457

Zhang J, Nagai C, Yu Q, Pan YB, Ayala-Silva T, Schnell RJ, Comstock JC, Arumuganathan AK, Ming R (2012) Genome size variation in three Saccharum species. Euphytica. 185:511-519

Zhang J, Zhang X, Tang H et al (2018) Allele-defined genome of the autopolyploid sugarcane Saccharum spontaneum L. Nat Genet

Publisher's Note Springer Nature remains neutral with regard to jurisdictional claims in published maps and institutional affiliations.

\section{Affiliations}

\section{Jinju Wei ${ }^{1} \cdot$ Zhihui Xiu $^{2}$ (D) Huiping Ou ${ }^{1}$. Junhui Chen ${ }^{2} \cdot$ Huayan Jiang ${ }^{2} \cdot$ Xiaoqiu Zhang ${ }^{1} \cdot$ Ronghua Zhang $^{1}$. Hui Zhou ${ }^{1}$ - Yiyun Gui ${ }^{1} \cdot$ Haibi $\mathrm{Li}^{1} \cdot$ Yangrui $\mathrm{Li}^{1} \cdot{ }^{1}$ Rongzhong Yang ${ }^{1} \cdot$ Dongliang Huang ${ }^{1} \cdot$ Hongwei Tan ${ }^{1} \cdot$ Xihui Liu $^{1}$}

1 Key Laboratory of Sugarcane Biotechnology and Genetic Improvement (Guangxi), Guangxi Key Laboratory of Sugarcane Genetic Improvement, Ministry of Agriculture, Sugarcane Research Center, Chinese Academy of Agricultural Sciences, Sugarcane
Research Institute, Guangxi Academy of Agricultural Sciences, Nanning 530007, China

2 BGI Genomics, BGI-Shenzhen, Shenzhen 518083, China 